\title{
SLOW-FAST DYNAMICS IN WONDERLAND
}

Alexandra Milik, Alexia Prskawetz, Gustav Feichtinger Institute for Econometrics, Operations Research and Systems Theory Vienna University of Technology, Vienna, Austria

Warren C. Sanderson

International Institute for Applied Systems Analysis

Laxenburg, Austria

RR-96-20

December 1996

Reprinted from Environmental Modeling and Assessment, Volume 1,2 (1996) pp. 3-17 [ISSN 1420-2026]. 
Research Reports, which record research conducted at IIASA, are independently reviewed before publication. Views or opinions expressed herein do not necessarily represent those of the Institute, its National Member Organizations, or other organizations supporting the work.

Reprinted with permission from Environmental Modeling and Assessment, Volume 1, No. 1,2 (1996) pp. 3-17 [ISSN 1420-2026].

Copyright (C)1996 Baltzer Science Publishers BV.

All rights reserved. No part of this publication may be reproduced or transmitted in any form or by any means, electronic or mechanical, including photocopy, recording, or any information storage or retrieval system, without permission in writing from the copyright holder.

Printed by Novographic, Vienna, Austria. 


\section{Foreword}

The Wonderland model is, in a way, a by-product of IIASA's work on understanding the nature of population-development-environment (PDE) interactions in different countries (so far Mauritius, Cape Verde, Yucatán, Botswana, and Namibia) and regions around the world. The true mechanisms by which these three factors interact are so complex and contextdependent that we decided to abstain from building a large global model and rather concentrate on specific settings. But whenever one does empirical work on a specific place and develops scenarios for possible future PDE interactions, one needs some type of stylized model to structure one's thinking. Many stylized models available are highly simplistic and static. The stylized model proposed by Warren Sanderson soon after the beginning of IIASA's PDE work went several steps further in dynamically linking the three factors.

Soon after its birth, this simple dynamic model, which was called "Wonderland" by Sanderson, developed its own dynamism. It was picked up by a group of scientists associated with Gustav Feichtinger at the Technical University of Vienna working on operations research and systems theory. The Wonderland model turns out to have several highly interesting nonlinear properties that make it attractive to scientists working in the forefront of nonlinear systems research as well as dynamic computer graphics. Although rather abstract, the model is not completely detached from empirical knowledge, and is being studied in an increasing number of countries. Recently we learned that a young Mexican statistician has entitled her master's thesis "A Wonderland Future for Mexico."

IIASA will continue its empirical and policy-relevant PDE analyses of specific regions and collaboration with the mathematically oriented modeling community. The Wonderland model shows that such cross-fertilization can be very productive.

Wolfgang Lutz Leader

Population Project 


\title{
Slow-fast dynamics in Wonderland ${ }^{*, * *}$
}

\author{
Alexandra Milik ${ }^{a}$, Alexia Prskawetz ${ }^{a}$, Gustav Feichtinger ${ }^{a}$ and Warren C. Sanderson ${ }^{b}$ \\ ${ }^{a}$ Institute for Econometrics, Operations Research and Systems Theory, Vienna University of Technology, \\ Argentinierstrasse 8, A-1040 Vienna, Austria \\ E-mail:alexia@e119ws1.tuwien.ac.at \\ b International Institute for Applied Systems Analysis, A-2361 Laxenburg, Austria, \\ and Department of Economics, State University of New York, Stony Brook, NY11794, USA
}

Received 18 October 1995; revised 9 February 1996

\begin{abstract}
Taking Wonderland - a simple model of demographic, economic and environmental interactions - as our artificial world, we illustrate the use of geometric singular perturbation theory in environmental demoeconomics. The theory of slow-fast dynamics helps us to gain new insights into the system's behaviour and enables one to reduce the inherent unpredictability of a "natural catastrophe" in Wonderland. Though we cannot predict the exact date of such an "environmental crash", we can state the specific demographic, economic and environmental constellations of our artificial world at which the sustainability of nature becomes endangered.
\end{abstract}

Keywords: Slow-fast dynamics, sustainability, population-development-environment.

\section{Introduction}

"One of the most important challenges facing mankind today is the formulation of strategies for sustainable development. ..." (Sanderson [12, p.8]) In spite of this obvious need for strategies to sustain the environment "models of environmental, demographic, and economic interactions have had frightfully short lifetimes, and typically, no descendants." (Sanderson [12, p.8])

Taking this challenge of investigating and understanding strategies for sustainable development and increasing the lifetimes of existing models, we aim to introduce a new mathematical approach to environmental population economics using Sanderson's Wonderland model (Sanderson [13]). In terms of its number of equations, Wonderland can be regarded as one of the simplest models of demographic, economic and environmental interactions to study the sustainability question. Though there are no structural changes in the model and all functions are deterministic, i.e. no stochastic forces are introduced, the system dynamics are unpredictable. In particular, many decades of common demographic and economic history do not necessarily imply even roughly similar demoeconomic futures. The reason being that Wonderland reacts nonlinearly to pollution flows.

A special feature of Wonderland (and ecological systems in general) is the fact that not all system variables

- The authors acknowledge financial support from the Austrian Science Foundation under contract No. P9608-SOZ.

** In particular the authors thank J. Cohen, L. MacKellar T. Muench, P. Szmolyan and F. Wirl for their helpful comments and explanations. evolve with the same velocity. The resulting mixture of slow and fast dynamics can lead to unpredictable, catastrophic transitions.

One main aim of this paper is to introduce the concept of geometric singular perturbation theory to a broader audience with focus on environmental issues. For this purpose we select Wonderland as our "artificial world" since it exactly fits our requirements: (1) it contains variables, which evolve with different velocities, (2) all equations are deterministic and (3) sustainability of the environment is the ultimate goal.

The paper is organized as follows. In section 2 we present the model and its assumptions. Section 3 gives a first insight into the system's dynamics. Its slow-fast behaviour suggests the use of concepts of geometric singular perturbation theory. The analysis of Wonderland in terms of slow-fast dynamics is presented in section 4 . The discussion of the main results obtained by means of geometric singular perturbation theory are presented in section 5 . We close with some conclusions and suggestions for further research (section 6).

\section{The model}

The world we shall step into now is Sanderson's "Wonderland" model of economic, demographic and environmental growth. How these three interact depends on the functional relationships assumed and the model parameters.

The original Wonderland model was written in discrete time. To facilitate the analysis of our artificial world in terms of slow-fast dynamics we change from discrete to continuous time steps. This modification 
poses no difficulties since the discrete version of Wonderland exhibits quite continuous time paths. Besides this change in the time concept, the model is exactly the same as in Sanderson [13].

The dynamics in Wonderland are characterized by four state variables:

$\boldsymbol{x}(\boldsymbol{t})$ population,

$\boldsymbol{y}(\boldsymbol{t})$ per capita output,

$z(t)$ quality of environment (natural capital),

$p(t)$ pollution per unit of output,

which evolve according to (for notational convenience we omit the time argument $t$ in the following)

$$
\begin{gathered}
d x / d t=x n(y, z), \\
d y / d t=y\left[\gamma-(\gamma+\eta)(1-z)^{\lambda}\right], \\
d z / d t=\nu z(1-z)\left[e^{\omega\left((\delta / \omega) z^{\rho}-f(x, y, z, p)\right)}-1\right], \\
d p / d t=-\chi p,
\end{gathered}
$$

where

$$
\begin{array}{lr}
c(y, z)=\varphi(1-z)^{\mu} y & \text { pollution control } \\
\bar{y}(y, z)=y-c(y, z) & \text { net per capita output }
\end{array}
$$

$f(x, y, z, p)=p x y-\kappa\left[\frac{e^{\sigma c(y, z) x}}{1+e^{\sigma c(y, z) x}}-0.5\right]$ pollution flow

$$
b(y, z)=\beta_{1}\left[\beta_{2}-\frac{e^{\beta \bar{y}(y, z)}}{1+e^{\beta \bar{y}(y, z)}}\right] \quad \text { crude birth rate }
$$

$d(y, z)=\delta_{1}\left[\delta_{2}-\frac{e^{\alpha \bar{y}(y, z)}}{1+e^{\alpha \bar{y}(y, z)}}\right]$

$\times\left(1+\delta_{3}(1-z)^{\vartheta}\right)$

crude death rate

$n(y, z)=b(y, z)-d(y, z) \quad$ population growth rate

with 20 parameters, which can be grouped as follows:

population: $\beta_{1}, \beta_{2}, \beta, \delta_{1}, \delta_{2}, \delta_{3}, \alpha, \vartheta$;

economy: $\gamma, \eta, \lambda$;

environment: $\quad \kappa, \sigma, \delta, \rho, \omega, \nu$;

environmental policy: $\varphi, \mu, \chi$.

Equation (1) states that population growth $n(y, z)$ depends endogenously on per capita output $y$ and the level of natural capital $z$. "The stock of natural capital may be thought of as the set of things provided to us by the environment, like air and water, which allow us to live healthy and productive lives." (Sanderson [13, p. 5]) Natural capital is assumed to be bounded in the interval $[0,1]$. If natural capital is not polluted at all, it takes on the value $z=1$. On the other extreme, when the environment is so polluted, that it produces the maximum possible damage to human health and to the economy, $z=0$.

The endogenous population growth rate itself is defined by the difference between the crude birth rate ( $b$; the ratio of births to the population) and the crude death rate ( $d$; the ratio of deaths to the population). It is assumed, that both crude rates decrease with increases in net per capita output $\bar{y}(y, z)$. Additionally, death rates rise as the stock of natural capital decreases.

Net per capita output $\bar{y}(y, z)$ is defined as per capita output, net of per capita expenditures on pollution control. Pollution control $c(y, z)$ in turn is determined by per capita output and the stock of natural capital. Per capita spending on pollution increases with per capita output and decreases with the stock of natural capital.

The availability of natural capital also influences the growth rate of the economy as indicated by equation (2). The lower the stock of natural capital, the lower will be the rate of per capita output growth. When the environment is totally polluted, i.e. $z=0$, per capita output shrinks at the rate $-\eta$, while per capita output increases at the rate $\gamma$ if environment is not polluted at all, i.e. $z=1$.

The growth of natural capital is assumed to be logistic. The speed at which natural capital regenerates (indicated by the term $\left.\nu\left[e^{\omega\left((\delta / \omega) z^{\rho}-f(x, y, z, p)\right)}-1\right]\right)$ depends positively on the level of natural capital $z$ and is negatively influenced by the amount of pollution $f(x, y, z, p)$, while $\nu$ represents a positive scaling factor. This specification is based on the idea that nature has the ability to cleanse itself, but that the strength of this ability diminishes as the stock of natural capital decreases. The function $g(z)=(\delta / \omega) z^{\rho}$ transforms the stock of natural capital into a flow of cleansing services measured in the same units as the pollution flow. When $\delta, \omega$ and $\rho$ are all positive, as they are in the Wonderland model, the cleansing flow diminishes as the stock of natural capital decreases. The difference between the two flows, $\left[(\delta / \omega) z^{p}-f(x, y, z, p)\right]$ is the net effect of natural and human forces on the environment. When the net flow is zero, the level of natural capital remains constant. The pollution flow that sets the net flow equal to zero, and which, therefore, maintains a constant stock of natural capital, is called the critical pollution flow. Clearly, the critical pollution flow is $(\delta / \omega) z^{\rho}$. If the actual amount of pollution, $f(x, y, z, p)$, is above the critical flow the environment deteriorates. If it is below the critical flow the environment regenerates. The fraction $\delta / \omega$ is the level of the critical flow when the environment is unpolluted $(z=1)$. The parameter $\rho$ determines how quickly the critical flow decreases as the stock of natural capital falls.

The flow of pollution $f(x, y, z, p)$ is determined by the impact on resources and the environment $p x y$ and the amount of pollution control $c(y, z)$. The term $p x y$ corresponds to the well known I-PAT identity (see e.g. Ehrlich and Holdren [2]), which states that the impact on natural resources and the environment, $I$, is related to 
the size of the population, $P$, to per capita output (affluence), $A$, and to technology, $T$, which refers to pollution generated per unit of output. If the environment is not polluted at all, the second term vanishes such that the pollution flow equals the I-PAT identity. On the other hand, if the environment is totally polluted, $z=0$, pollution control is at its maximum.

We have to make two points of clarification about equation (3). First, we used the term "pollution flow" to refer to entrance of new pollutants into the environment. Another term for pollution flow is emissions. Pollution flows cumulate into pollution stocks. For simplicity, though, these stocks are omitted from the model. How polluted the environment is can be determined by the value $z$. The lower the value of $z$, the more polluted is the environment. Pollution control expenditures are assumed to depend on how polluted the environment is (i.e. on $z$ ) and not on the current flow of emissions. For example, we spend money on reducing the amount of particulate matter in the air because the environment is polluted and we have difficulty breathing. If we lived in a place where the wind always blew the particles away and we were left always with clean air, we would not spend any money on pollution control, even though there were pollution flows.

Finally equation (4) represents an exogenously changing technology. In each time period pollution per unit of output is assumed to decrease at a constant rate $\chi$.

Wonderland is reminiscent in a certain sense of the World 3 model that was the basis of the book The Limits to Growth (Meadows et al. [8]). There are important differences, however. The World 3 model was used to claim that the world was on an unsustainable path and that if we remained on that path the world would experience an environmental crash. The structure of the World 3 model guaranteed that it would crash (see Sanderson [13]). The Wonderland model is not designed to represent the entire world or to provide morals about where we are going. The Wonderland model can exhibit crashes and perpetual growth. It is the condition which differentiates perpetual growth from environmental crash that concerns us.

Summing up, Wonderland is characterized by three endogenously interacting modules; the population $x$, the economy $y$ and the environment $z$. The positive and negative feedbacks together with the nonlinearity of the functional relationships will turn out to be responsible for the seemingly unpredictable future in Wonderland. All together, Wonderland promises to be an interesting world to study and to understand the complex interactions between population, the economy and the environment observed in reality.

\section{Numerical results}

We first repeat two of the simulation results given in San- derson [14] using the continuous analogue of Wonderland presented in the previous section. The numerical calculations have been performed using the LOCalBIFurcation program LOCBIF (Khibnik et al. [5]). For numerical integration we used a stiff fifths-order solver.

Figure 1 demonstrates the future of Wonderland if pollution per unit of output decreases at the rate $\chi=0.03$. The other parameters are just the same as given in Sanderson [14]:

Population: $\beta_{1}=0.04, \beta_{2}=1.375, \beta=0.16, \delta_{1}=0.01$, $\delta_{2}=2.5, \delta_{3}=4, \alpha=0.18, \vartheta=15$;

Economy: $\gamma=0.02, \eta=0.1, \lambda=2$;

Environment: $\kappa=2, \sigma=0.02, \delta=1, \rho=2, \omega=0.1$, $\nu=1$;

Environmental policy: $\varphi=0.5, \mu=2$,

with the initial conditions set at the values: $x=y=p=1, z=0.98$.

In this case, Wonderland yields a sustainable future. Per capita output increases over time, population converges towards the stationary level of zero growth rate and the pollution flow steadily decreases - after a short initial phase of rising pollution - such that natural capital can be maintained.

When $\chi$ is reduced from 0.03 to 0.01 , the economists' dream turns into the environmentalists' nightmare (figure 2). Now Wonderland no longer obeys the criterion of sustainability. The reason being that pollution accumulates over time compared to the declining pollution flow in the economists' dream scenario. All of a sudden natural capital decreases followed by a decrease in per capita output and population.

We add an additional scenario (figure 3 ) not yet found in Sanderson [13] in which we assume a much more cost-effective pollution control technology (we increase $\kappa$ from 2 to 100 ) but retain all the other parameters of the environmentalists' nightmare scenario. Compared to figure 2, the pollution flow declines much faster once natural capital falls below 1 . If $\kappa$ is large enough the pollution flow might even become negative (see figures 11a, b; section 5) meaning that the environment is made cleaner than it would have been even without pollution. As is illustrated in figure 3 , a very effective pollution control renders a recovering of the environment possible.

In figure 4, these three scenarios are illustrated in a three-dimensional phase space. The unpredictability of the future in Wonderland becomes visible. Years of common demographic and economic history do not necessarily imply roughly similar demoeconomic futures. All of a sudden, these three scenarios - differing in the assumption of the exogenous given production technology and the effectiveness of pollution control-diverge.

Now the question arises what are the preconditions (in terms of the system's dynamics) for the sudden envir- 

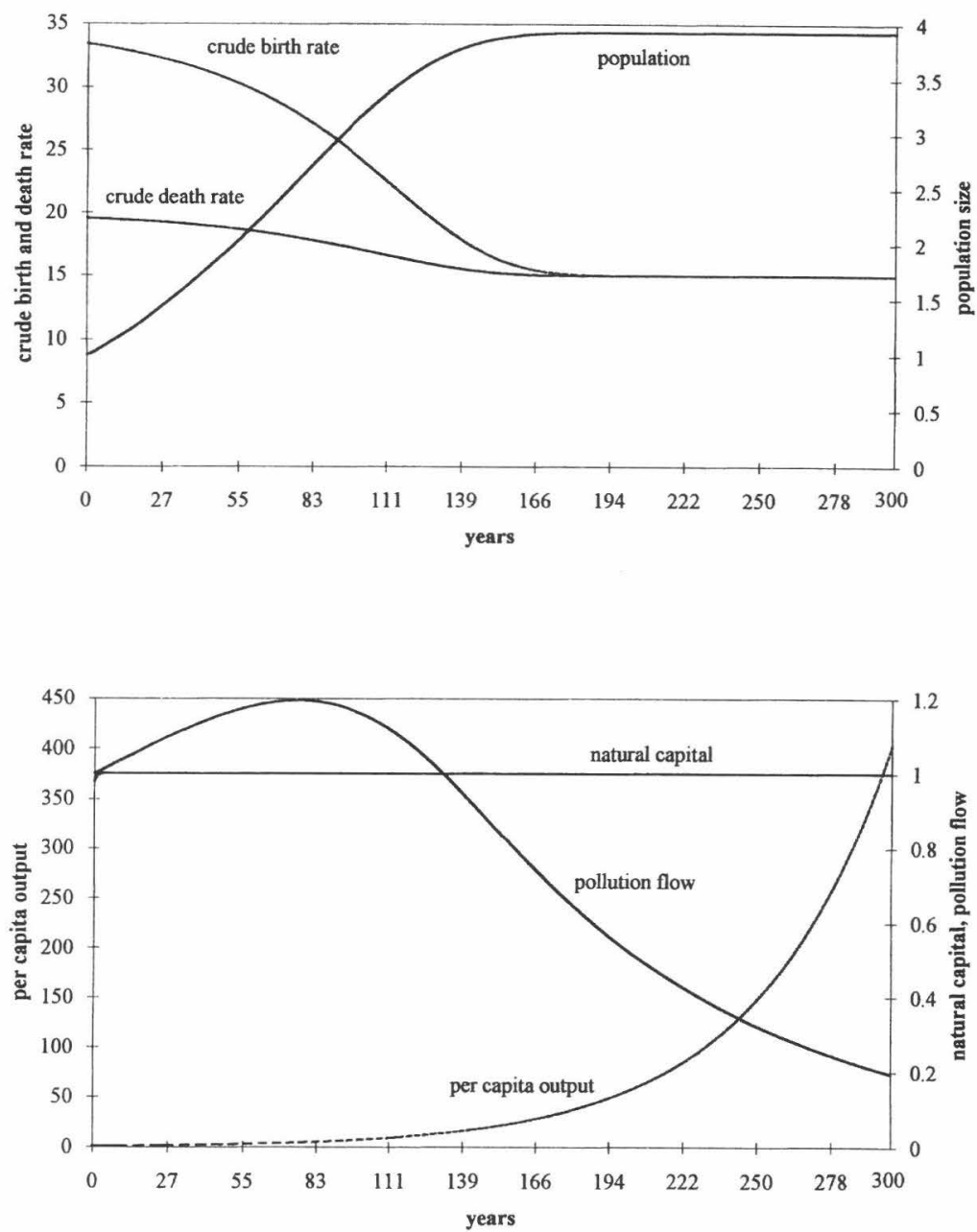

Figure 1. Economists' dream scenario; $\chi=0.03$.

onmental crash in Wonderland. Of course, decreasing the technology parameter $\chi$ results in higher pollution flows. But why does environmental degradation happen all of a sudden and not continuously over time, which would certainly increase the ability to adjust to the changing environment before the actual crash occurs.

For a first insight into the system's behaviour we omit the dynamics of the pollution per unit of output at the moment and consider it as a parameter. Hence, we assume constant technologies of pollution abatement $\chi=0$, which result in a constant value of $p=1$. The other parameters are just the same as given in figure 1 .

Figure 5 shows the three dimensional flow in phase space for several initial conditions. We observe that the system variables change with very different velocities, i.e. they exhibit slow-fast dynamics. Consider the initial point $x=1, y=1, z=0.5$, then the quality of the environment $z$ increases very fast to a high level while population $x$ and output $y$ stay nearly constant. After this phase of fast evolution, output and population increase with a slow speed. The environment stays on its high level until output (and consequently pollution) is too high and environmental quality drops very fast. This "environmental collapse" stops at a very low value near $z=0$ and is followed by a slow decline of population and the economy.

\section{Analysis of the model in terms of slow-fast dynamics}

Differential equations of slow-fast systems can usually 

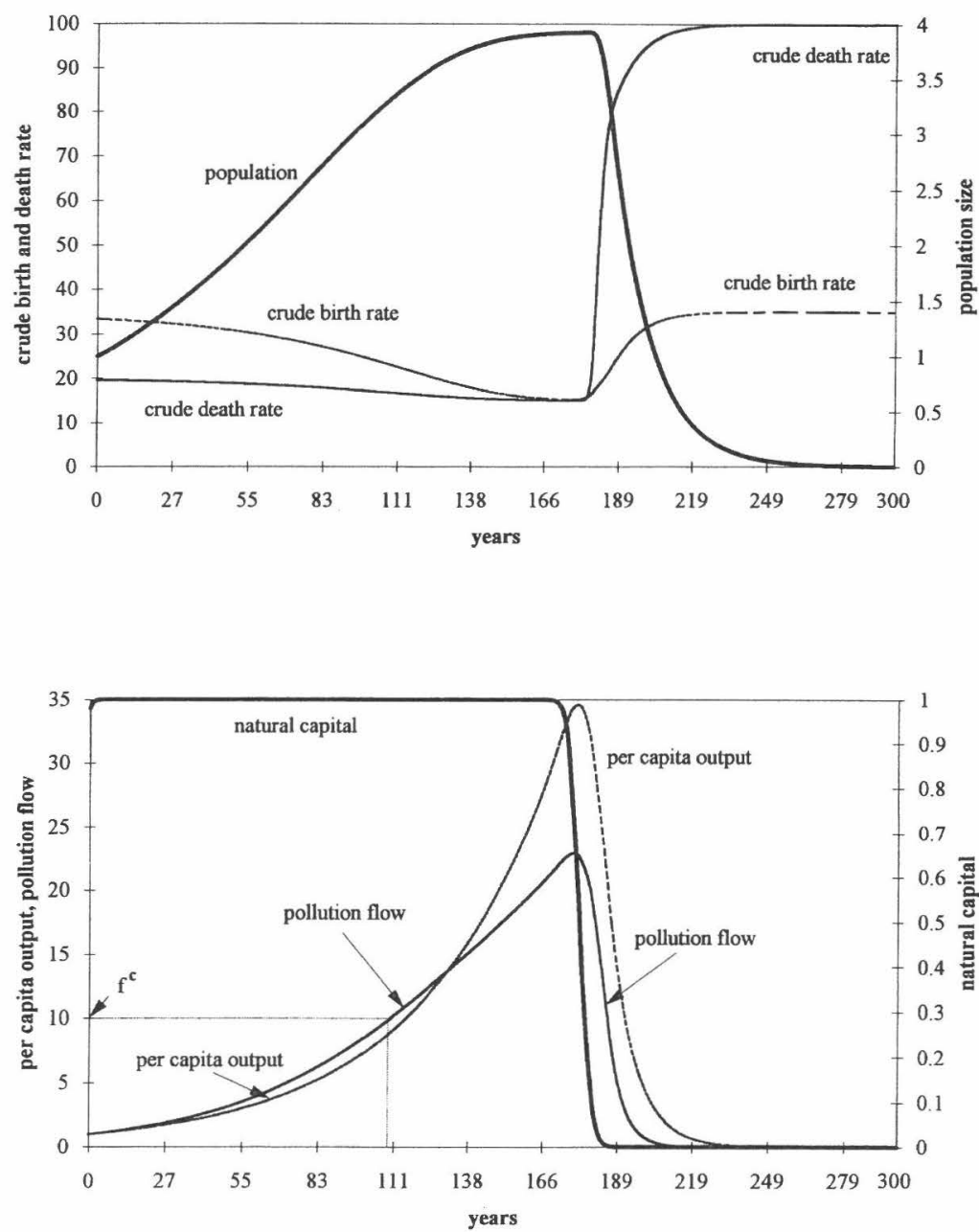

Figure 2. Environmentalists' nightmare scenario; $\chi=0.01$. The letter $f^{c}$ indicates the critical level of pollution flow for $z=1$

be scaled, such that one or more of the dynamic laws of the state variables is multiplied by a small parameter. Then the small parameter can be regarded as perturbation parameter and the differential equations can be studied in the framework of singular perturbation theory. Classically this involves asymptotic expansions in the perturbation parameter and gives quantitative results for single solutions within a certain time horizon [9]. But here we are interested in the qualitative behaviour of the system, i.e in the geometry of the orbits in phase space, rather than the time paths of the system's dynamics. Therefore we analyze the model using concepts of geometric singular perturbation theory. This theory goes back to Fenichel [3] who uses the geometric concepts of dynamical systems theory to analyze singularly perturbed systems of differential equations. For an account of this theory the reader is referred to Arnol'd [1] or Szmolyan [16] who also gives some interesting applications. For applications in population dynamics see Rinaldi and Muratori [10,11]. Unfortunately the functions in our model are too complicated to do such an analysis in a completely rigorous manner. However, combining the basic geometric ideas of the theory with numerical simulation yields new insights into the dynamics of Wonderland.

First we investigate which property of the equations of motion causes the slow-fast behaviour of the system. The orders of magnitude of the rate of change (i.e. of the speed) are determined by the parameters $\nu=1$ for the variable $z, \beta_{1}=0.04$ and $\delta_{1}=0.01$ for the variable $x$ and $\gamma=0.02$ and $\eta=0.1$ for the variable $y$. To express the difference in the order of magnitude of the parameter 

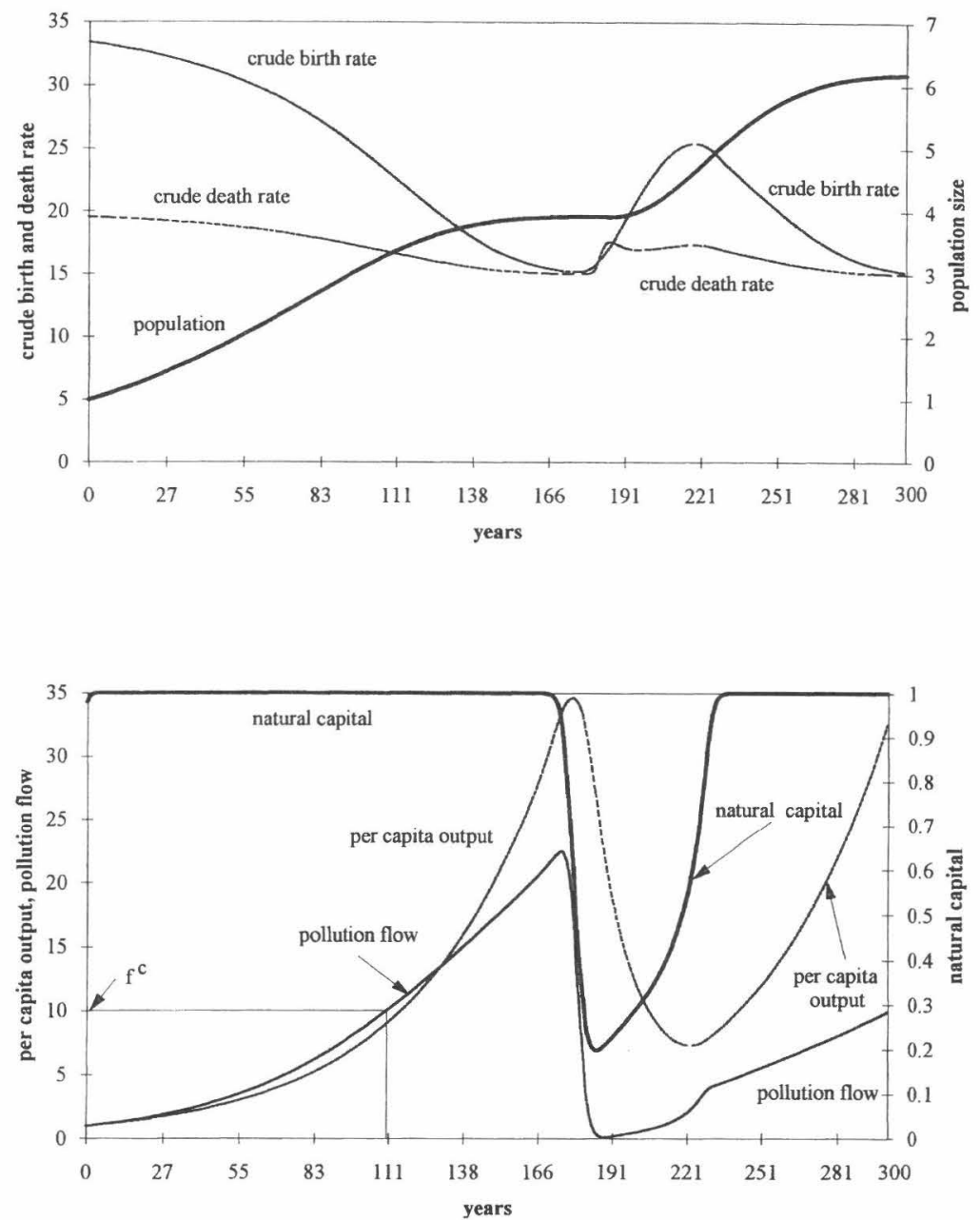

Figure 3. Escape from environmental catastrophe; $\chi=0.01, \kappa=100$. The letter $f^{c}$ indicates the critical level of pollution flow for $z=1$.

values we define a small parameter $0<\varepsilon \ll 1$ and rescale the parameter $\nu$ according to

$$
\nu \rightarrow \frac{\nu}{\varepsilon}
$$

With our choice of parameters $\varepsilon=0.01$ is a reasonable value and the system can be written as

$$
\begin{gathered}
d x / d t=x n(y, z), \\
d y / d t=y\left[\gamma-(\gamma+\eta)(1-z)^{\lambda}\right], \\
\varepsilon d z / d t=\nu z(1-z)\left[e^{\omega\left((\delta / \omega) z^{\rho}-f(x, y, z, p)\right)}-1\right] .
\end{gathered}
$$

In this rescaled form the system's slow-fast behaviour is more obvious. Unless the right hand side of the equa- tion for $z$ is not small $z$ evolves much faster than $x$ and $y$ since the rate of change of the variable $z$ is multiplied by $1 / \varepsilon$ and $\varepsilon$ is small. System (6) is in the standard form of singular perturbation theory where $\varepsilon$ is the perturbation parameter. Here time is measured on the scale of the slow variables $x$ and $y$ and we have $t \in[0, T]$ with a finite time horizon $T$.

We may write the system as well in the time scale of the fast variable $z$. We rescale time by $t \rightarrow \tau \varepsilon$ and obtain

$$
\begin{gathered}
d x / d \tau=\operatorname{\varepsilon xn}(y, z), \\
d y / d \tau=\varepsilon y\left[\gamma-(\gamma+\eta)(1-z)^{\lambda}\right],
\end{gathered}
$$




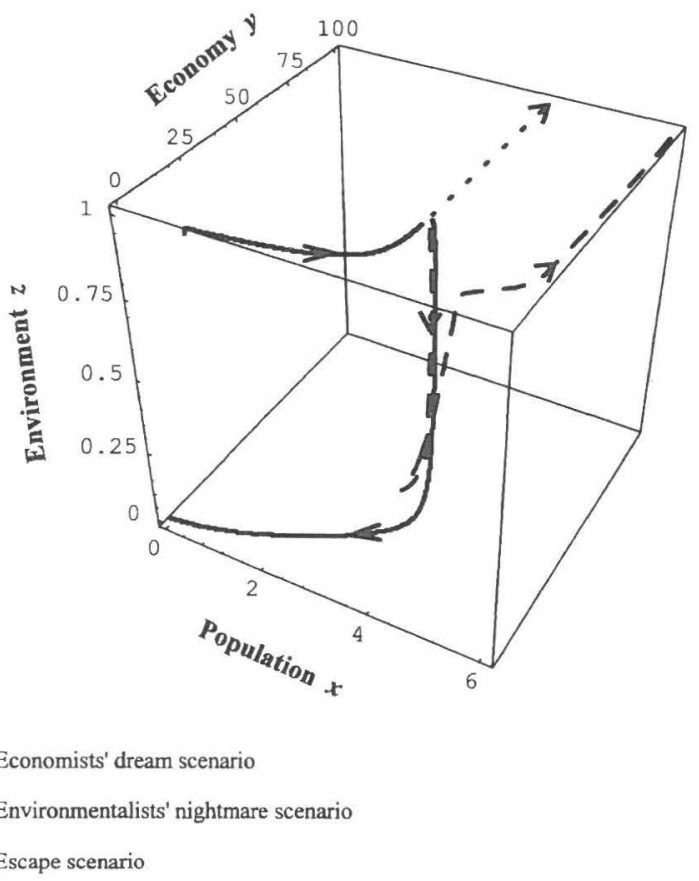

Figure 4. Economists' dream, environmentalists' nightmare and escape scenario in phase space.

$$
d z / d \tau=\nu z(1-z)\left[e^{\omega\left((\delta / \omega) z^{\rho}-f(x, y, z, p)\right)}-1\right],
$$

with $\tau \in[0, T / \varepsilon]$.

For $E>0$ it is equivalent to the slow system (8) whereas in the limit $\varepsilon \rightarrow 0$ we obtain two essentially different systems, the reduced system

$$
\begin{gathered}
d x / d t=x n(y, z), \\
d y / d t=y\left[\gamma-(\gamma+\eta)(1-z)^{\lambda}\right], \\
0=\nu z(1-z)\left[e^{\omega\left((\delta / \omega) z^{\rho}-f(x, y, z, p)\right)}-1\right],
\end{gathered}
$$

and the layer problem

$$
\begin{gathered}
d x / d \tau=0, \\
d y / d \tau=0, \\
d z / d \tau=\nu z(1-z)\left[e^{\omega\left((\delta / \omega) z^{\rho}-f(x, y, z, p)\right)}-1\right] .
\end{gathered}
$$

Both systems capture some aspects of the dynamics but with limited validity in phase space and time. However, one may arrive at a global picture of the dynamics by matching the solutions of these different systems. To show that matching the solution in phase space is possible is all what geometric singular perturbation theory is about. Therefore we formulate the problem in the geometric language of dynamical systems theory.
The layer problem (11) is seen as a one dimensional dynamical system for the variable $z$ with the slow variables $x$ and $y$ acting as parameters. We are interested in the dependence of equilibrium solutions of system (11) on $x$ and $y$. The equilibria of the system are $z=0$ and $z=1$ for all $(x, y) \in \mathbb{R}^{2}$ and $z=z(x, y)$ which is defined implicitly as the solution of the equation

$$
\frac{\delta}{\omega} z^{\rho}-f(x, y, z, p)=0
$$

This equation can not be solved analytically. However, we can visualize the set of equilibria of the layer problem with numerical computations using the program package Mathematica (Wolfram [17]). The set of equilibria consists of three smooth manifolds $Z_{r}, Z_{0}$ and $Z_{1}$ defined as the graphs $z=z(x, y)$ for all $(x, y) \in U \subset \mathbb{R}^{2}, z=0$ for all $(x, y) \in \mathbb{R}^{2}$ and $z=1$ for all $(x, y) \in \mathbb{R}^{2}$. Figure 6 shows these manifolds which in our case are surfaces in the three-dimensional space $x, y$, $z$. The surface $Z_{r}$ intersects $Z_{0}$ in the $x$ and $y$ axes and intersects $Z_{1}$ in a curve $C$ given by the solution of equation (12) with $z=1$ :

$$
x y p=\frac{\delta}{\omega} .
$$

In the following we use the terms critical surface and critical curve for $Z_{r}$ and $C$ respectively.

Generally the local stability of an equilibrium is deter- 


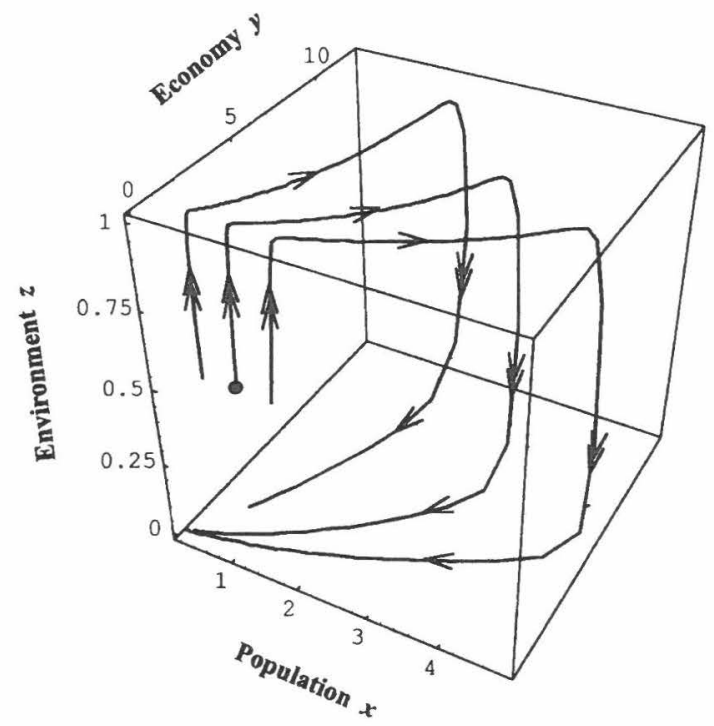

$\longrightarrow$ fast dynamics

$\longrightarrow$ slow dynamics

Figure 5. Trajectories in phase space for several initial conditions.

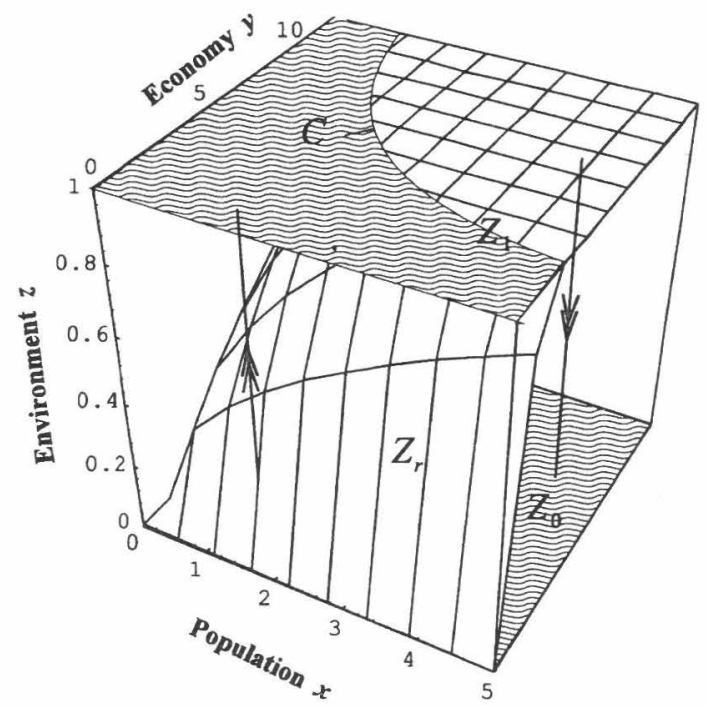

attracting manifold

repelling manifold

Figure 6. Slow manifolds $Z_{0}, Z_{1}$ and $Z_{\mathrm{r}}$ and orbits of the layer problem. 
mined by the sign of the real part of the eigenvalues of the Jacobian evaluated at the equilibrium. In our case the Jacobian is simply the derivative of the right hand side in equation (11). By calculating the sign of this derivative at all points of the three surfaces of equilibria $Z_{1}$, $Z_{0}$ and $Z_{r}$ we obtain the following results: The two surfaces of equilibria $Z_{1}$ and $Z_{0}$ are attracting for $x y p<\delta / \omega$ with their basins of attraction separated by the repelling critical surface $Z_{r}$ given by equation (12) while for $x y p>\delta / \omega, Z_{1}$ is repelling and only $Z_{0}$ is attracting (see figure 6). For the equilibria on the critical curve $C$ given by $x y p=\delta / \omega$ the Jacobian is zero and therefore the equilibria on this curve are nonhyperbolic.

The implicit equation (12) for the repelling critical surface $Z_{r}$ plays a crucial role for the system. If we choose initial conditions for which $f(x, y, z, p)$ is less than

$$
\frac{\delta}{\omega} z^{\rho}
$$

the trajectory is attracted by the surface of equilibria $Z_{1}$, otherwise it is attracted by $Z_{0}$.

Now let us analyze the reduced problem (8)-(10) in terms of dynamical systems theory. It is a two dimensional dynamical system for the variables $x$ and $y$ on the solution set of (10). This set is precisely the set of equilibria of the layer problem and consists of the three sur- faces $Z_{0}, Z_{1}$ and $Z_{r}$. In geometric singular perturbation theory they are called the slow manifolds or slow surfaces. They connect the qualitative dynamics of the layer problem and the reduced problem.

On the slow surface $Z_{1}$ the reduced problem reads

$$
\begin{gathered}
d x / d t=x n(y, 1), \\
d y / d t=y \gamma,
\end{gathered}
$$

and on $Z_{0}$

$$
\begin{gathered}
d x / d t=x n(y, 0), \\
d y / d t=-\eta y .
\end{gathered}
$$

Figure 7 shows numerically obtained orbits of the reduced system on the slow surfaces $Z_{1}$ and $Z_{0}$. On $Z_{1}$ the slow variables $x$ and $y$ are increasing while on $Z_{0}$ both slow variables are decreasing.

Now, we combine the results on the two limiting problems which is possible for those parts of phase space where the slow surfaces $Z_{0}, Z_{1}$ and $Z_{r}$ are hyperbolic, i.e. away from the nonhyperbolic critical curve $C$ (hyperbolicity assumption). Then and for $\varepsilon$ sufficiently small all surfaces persist as locally invariant attracting or repelling slow surfaces $Z_{0}^{\varepsilon}, Z_{1}^{\varepsilon}$ and $Z_{r}^{\varepsilon}$ in the singularly perturbed problem (see Fenichel [3] or Szmolyan [16]). Thus changing the perturbation parameter $\varepsilon$ from zero to a

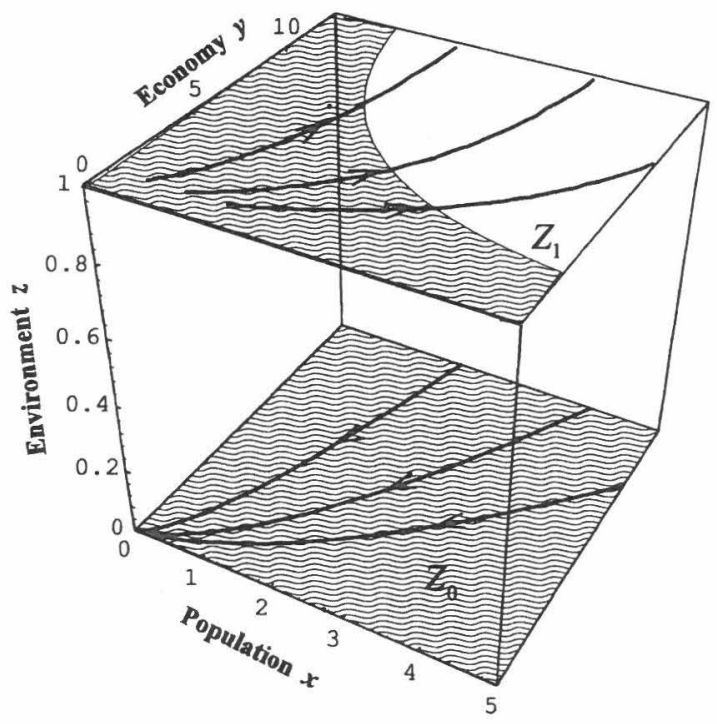

attracting manifold

$\longrightarrow$ orbit of reduced problem

Figure 7. Orbits of the reduced problem on the slow manifolds $Z_{0}$ and $Z_{1}$. 
small finite value does not destroy the main geometric objects which connect the qualitative dynamics of the layer problem and the reduced system. As a consequence the layer problem captures the fast movement of the variable $z$ towards or away from the slow manifolds while the reduced problem gives a good approximation of the slow evolution of $x$ and $y$ on the attracting manifold $Z_{0}$ or $Z_{1}$ (see figure 8 ).

Putting the pieces of this dynamic puzzle together gives the following description of the slow-fast behaviour of one trajectory. Consider the initial point $x=1$, $y=1, z=0.5$. The variable $z$ increases very fast according to the dynamics of the layer problem until it reaches the attracting surface of equilibria $Z_{1}$. During this short time interval the slow variables $x$ and $y$ stay almost constant. After this initial phase $x$ and $y$ increase slowly according to the reduced problem on the slow surface $Z_{1}$. When the product $x y$ reaches a level given by equation (13), i.e. the trajectory passes the critical curve $C$, the equilibrium manifold $Z_{1}$ loses its stability. This is a critical point where the hyperbolicity assumption for the slow surface is violated and the theory fails. Indeed, we observe an interesting and somewhat counterintuitive phenomenon around this point. The trajectory is not repelled from $Z_{1}$ immediately but follows closely the unstable part of the manifold $Z_{1}$ for a while until it is ulti- mately repelled away. It seems as if the slow-fast system needs some time to "feel" the change in stability. This is related to the exponential attractiveness of the stable part of the surface $Z_{1}$. The trajectory is exponentially close to the surface. Therefore it takes a considerable time interval $O(1)$ until the trajectory leaves a small $O(\varepsilon)$ neighbourhood of the now repelling surface. This phenomenon is not completely understood in a rigorous way, except in simple cases, see e.g. Schecter [15].

After the delay the variable $z$ drops very fast as it is described quite accurately by the dynamics of the layer problem. The trajectory is attracted to the second equilibrium surface $Z_{0}$ and follows again the slow dynamics described by the reduced system on the surface $Z_{0}$.

Until now we considered pollution per unit of output $p$ as a parameter. Whereas in the complete Wonderland model $p$ follows an independent dynamic given by

$$
d p / d t=-\chi p .
$$

To explain the solutions of the general equations with $\chi>0$ we can use the above results on the special case $\chi=0$. As $\chi$ is small, i.e. in the order of $\varepsilon, p$ is a slow variable and equation (17) does not change the layer problem. We only have to consider $p$ as a parameter for the layer problem. The main effect of $p$ is to influence the repelling surface $Z_{r}$ and, consequently, the curve $C$

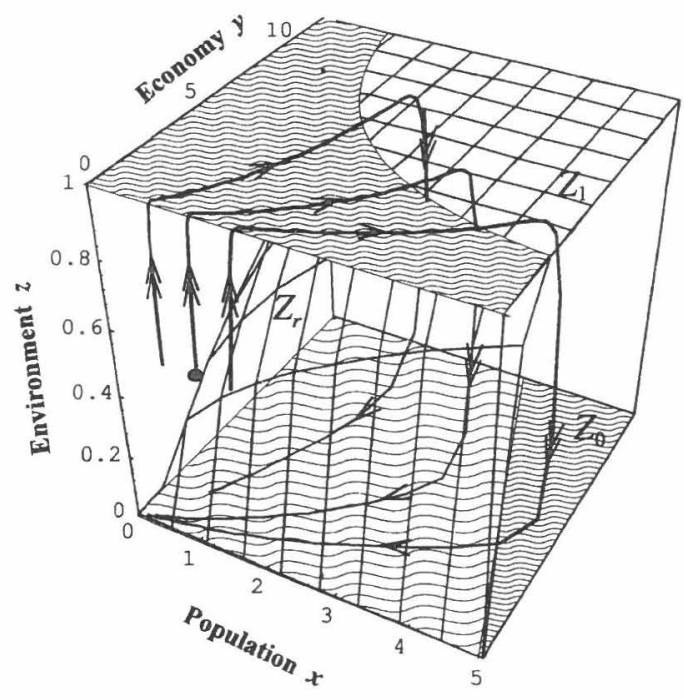

attracting manifold

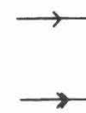

slow evolution of singularly perturbed system

repelling manifold

fast evolution of singularly perturbed system 
where the surface $Z_{1}$ loses its stability (see equations (12) and (13)).

Figure 9 shows how a decrease in $p$ as it is described by its slow dynamics moves the stability front on $Z_{1}$ to larger values of $x$ and $y$. But at the same time $x$ and $y$ also increase. The slow dynamics of the crucial product $x y p$ is given by

$$
x(t) y(t) p(t)=x(t) e^{(\gamma-\chi) t}
$$

with $0<x(t)<k \in R$. Thus for $\gamma<\chi$ the product is decreasing and the orbits always stay in the stable part of the surface $Z_{1}$. This corresponds to the economists' dream scenario. If $\gamma>\chi$ then $x y p$ is exponentially increasing and will inevitably exceed the value $\delta / \omega$. As in the static case $(\chi=0)$ the trajectory stays near the now unstable slow manifold for a while until it is ultimately attracted to the other slow manifold $Z_{0}$. This is the environmental crash of the environmentalists' nightmare scenario as illustrated in figure 2 .

\section{Discussion}

The use of the concepts of geometric singular perturbation theory together with the numerical calculations in the preceding section yield new insights into the system dynamics of Wonderland.

The manifold $Z_{r}$, which separates the economists' dream scenario (natural capital is not polluted at all, $z=1$ ) from the environmentalists' nightmare scenario (natural capital is totally polluted, $z=0$ ), essentially determines the future of Wonderland. Therefore, we shall denote this manifold environmental change frontier in the following. Equation (12) of the manifold $Z_{r}$ yields

$$
\frac{\delta}{\omega} z^{\rho}=f^{c}(x, y, z, p)
$$

where $f^{c}$ denotes the critical pollution flow that solves equation (19). The adjective critical is used to indicate the specific role of this repelling manifold $Z_{r}$ for the question of sustainability. In particular, if the actual pollution flow $f(x, y, z, p)$ exceeds the critical pollution flow, we are attracted to the manifold $Z_{0}$ implying that the environment deteriorates (natural capital decreases). Contrary, if the actual pollution flow is below the critical pollution flow, we are attracted to the stable part of the manifold $Z_{1}$ leading to an improvement of the environment (natural capital increases). If natural capital is not polluted at all, $z=1$, equation (19) reduces to

$$
\frac{\delta}{w}=f^{c}(x, y, 1, p)
$$

and the actual pollution flow $f(x, y, z, p)$ can be written as the well known I-PAT identity (see section 2)

$$
x y p=f(x, y, 1, p) .
$$

The impact on resources and the environment as measured by the pollution flow $f(x, y, 1, p)$ is related to the size of the population, $x$, to per capita output, $y$, and to technology, $p$, which refers to pollution generated per unit of output. Equation (20) simply states, that the "critical" impact on resources if natural capital is not polluted at all $(z=1)$, is solely determined by the parameters $\delta$ and $\omega$ which characterize the ability of the resources to cleanse itself.

Figures 10 and 11 show the critical, $f^{c}(x, y, z, p)$, and the actual, $f(x, y, z, p)$, pollution flow for the economists' dream scenario, the environmentalists' nightmare scenario and the escape scenario as already illustrated in section 3 .

In the economists' dream scenario (figure 10, upper part) the actual pollution flow never exceeds the critical pollution flow, so that sustainability of nature is guaranteed forever. In case of the environmentalists' nightmare scenario (figure 10, lower part) the actual pollution flow exceeds the critical pollution flow already at time point $T_{1}(=109)$. But surprisingly "'nothing" happens for quite a while; per capita output and population continue to grow and natural capital stays near its highest

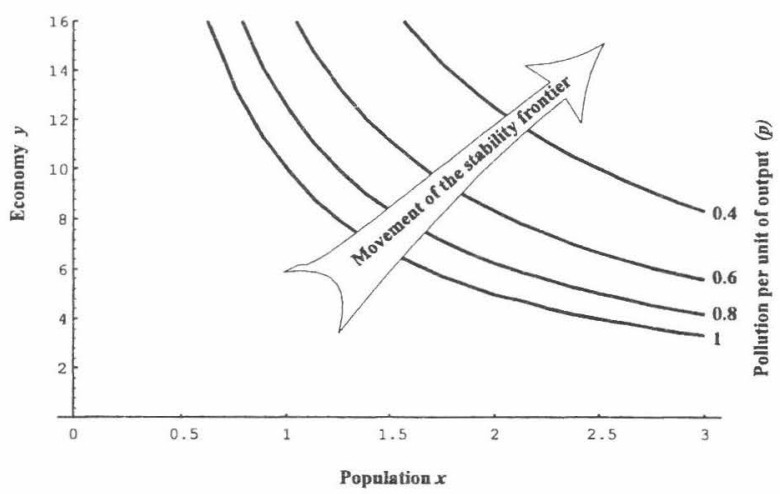

Figure 9. Movement of the stability frontier with decreasing pollution per unit of output. 

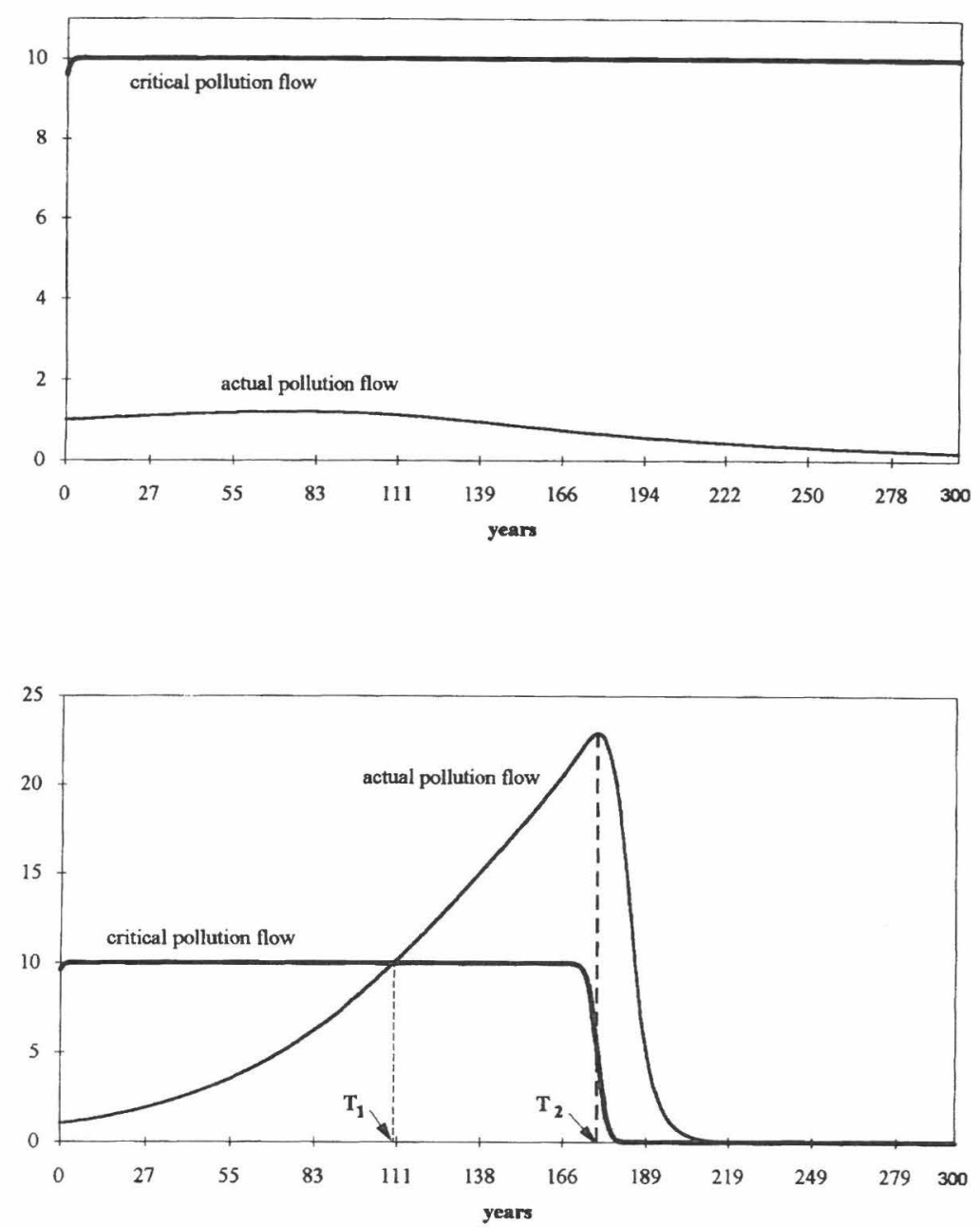

Figure 10. (Upper) Critical and actual pollution flow in the economists' dream scenario. (Lower) Critical and actual pollution flow in the environmentalists' nightmare scenario.

possible level (see figure 2). The environmental crash which we date as occurring when the critical flow $(\delta / \omega) z^{\rho}$ is one half - only happens at time point $T_{2}(=177), 68$ time steps after the critical pollution flow has been crossed. This point is discussed in more detail in Gragnani et al. [4]. This is very important for practical purposes, because noticeable changes may come only after the pollution flow is significantly above the critical value. Since the actual pollution flow always stays above the critical pollution flow from there on, the environment continually deteriorates and we end up with no natural capital being left, $z=0$. The escape scenario (figure 11) exhibits similar dynamics up to the point $T_{2}$, where the actual environmental crash occurs. But once natural capital decreases, the very cost-effective pollution control in the escape scenario (recall that we increase the parameter $\kappa$ from 2 to 100 , while we retain all the other parameters of the environmentalists' nightmare scenario) leads to a much faster decline in the actual pollution flow as compared to the environmentalists' nightmare scenario such that the critical pollution flow is crossed again. As soon as the actual pollution flow is below the critical pollution flow, natural capital can increase again. As it is shown in the lower part of figure 11, there exist several crossings of the critical pollution flow and natural capital oscillates over time. 

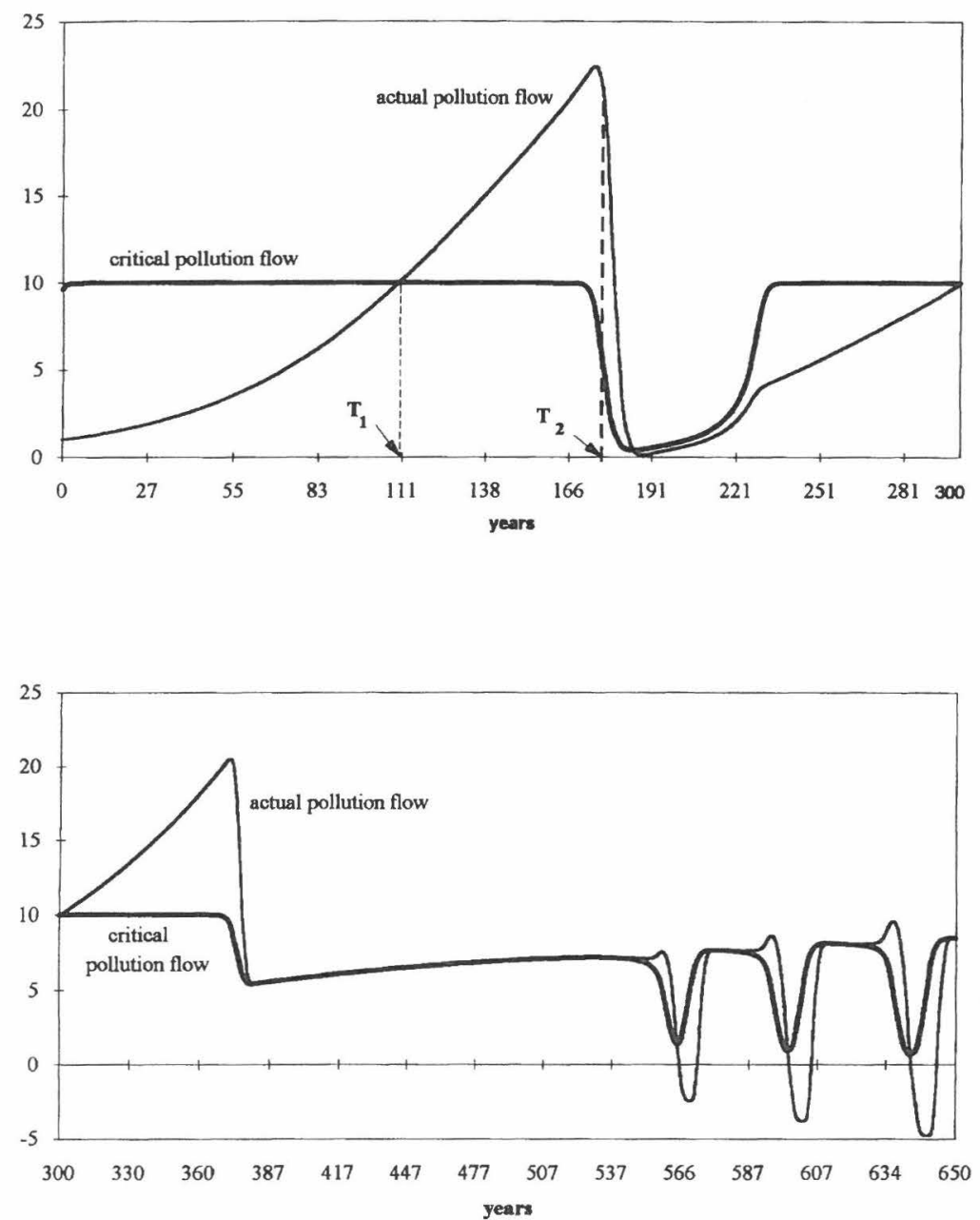

Figure 11. Critical and actual pollution flow in the escape scenario.

The following expression,

$$
x(t) e^{(\gamma-\chi) t}-\kappa\left[\frac{e^{\sigma c(y, z) x}}{1+e^{\sigma c(y, z) x}}-0.5\right],
$$

is an attainable upper bound of the pollution flow in time period $t$. To increase the possibility of sustainable development, we want this upper bound to be as small as possible. In particular, this upper bound decreases as $\chi$ and $\kappa$ increase and $\gamma$ decreases.

As long as the rate of decrease of pollution per unit of output, $\chi$, exceeds the growth of output, $\gamma$, we will never exceed the critical pollution flow. On the other extreme, if the rate of decrease of pollution per unit of output is equal to 0 , we will inevitably cross the border, since there is no other counterbalancing factor to the exponential growth of the economy, except the technology.

Now, assume that we have crossed the critical pollution flow. In this case, whether the actual pollution flow decreases fast enough such as to cross the critical pollution flow before the environment is totally depleted, will depend on the strength of human intervention (pollution control, which is represented by the second term of equation (22)).

Summing up, though we cannot predict the exact date of an environmental crash, we are able to state the demographic, economic and environmental constellations of our artificial world where the sustainability of 
nature becomes endangered, i.e. when we have crossed the limit. Moreover, we can explicitly state the conditions that prevent an environmental crash or at least renders a recovering of the environment possible once an environmental crash has occurred.

\section{Conclusions}

The starting point of the present paper are the mutual nonlinear demographic, economic and environmental interactions in an artificial world called "Wonderland". Central to the future of Wonderland is the question which constellations of the parameters and initial conditions foster sustainability and growth.

Though there are no stochastic, exogenous shocks, the future of Wonderland seems quite unpredictable. Sanderson [13] presents several simulation results ranging from the economists' dream scenario (increasing per capita output levels, stationary population levels and clean environment) to the environmentalists' nightmare scenario, where natural capital (air, water) is totally polluted. Amazingly, the "environmental catastrophe" seems to happen all of a sudden.

To explain these dynamics in Wonderland we introduce the powerful and flexible approach provided by phase portraits. Lee $[6,7]$ was one of the first who used phase diagram analysis to investigate the complex interactions of demographic and economic variables. In particular he suggested several further extensions of his model (Lee [7, p. 287]): “A fuller model would permit resource constraints to bound the system's eventual growth, .... Such a model would lead to a more pessimistic view of the consequences of population growth". In Wonderland these suggestions are taken up by incorporating natural capital as an additional system variable which interacts with population as well as economic growth.

A special feature of Wonderland is the fact that environmental, economic and demographic variables change with different velocities, i.e. they exhibit slow-fast dynamics. By making use of this property we are able to state the specific demographic, economic and environmental constellation of our artificial world where sustainability of nature begins to be endangered. As long as the rate of pollution decrease exceeds the growth rate of the economy, this border (denoted environmental change frontier) will not be crossed. The future will be sustainable in this case and there is no necessity for people to control pollution. But once the economic growth rate surpasses the rate of pollution decrease, the sustainability of Wonderland is endangered. Now, sustainability depends on human intervention. In particular, the strength of pollution control determines whether Wonderland ends up in a natural catastrophe (the envi- ronment is totally destroyed) or if we can get back to economic growth and sustainability.

Surprisingly, even when we have crossed the environmental change frontier, the decrease in natural capital, which will be unavoidable, might occur many years later. We only know, when we have crossed the limit, but we cannot predict when the environmental crash will actually happen. We might well live in growing prosperity for a long while (growing per capita output and population) after we have crossed the "border". This fact once more highlights the importance of environmental preservation to guarantee a sustainable future.

In any case, knowing the "critical" border and the specific tradeoff between economic growth and the pollution flow, increases the ability to adjust to the changing environment before the actual crash occurs.

Further investigation of the time it takes for environment to deteriorate after the environmental frontier has been crossed seems a promising avenue for future research. Including endogenous technology and capital accumulation, that is, studying higher order descriptions of economic, demographic and environmental interactions, may facilitate even more complex solutions. On the other hand, it might well lead to new insights into the interactions between the environment and the economic-demographic sector.

\section{References}

[1] V.I. Arnol'd, Dynamical Systems V. Encyclopedia of Mathematical Sciences 5, Springer, Berlin, 1994.

[2] P. Ehrlich and J. Holdren, Impact of population growth, Science 171 (1971) 1212-17.

[3] N. Fenichel, Geometric singular perturbation theory, Journal of Differential Equations 31 (1979) 53-98.

[4] A. Gragnani, A. Milik, A. Prskawetz and W.C. Sanderson, Persistent unstable equilibria in Wonderland, working paper WP95-118, IIASA, Laxenburg, 1995.

[5] A. Khibnik, Y. Kusznetsov, V. Levitin and E. Nikolaev, Continuation techniques and interactive software for bifurcation analysis of ODEs and iterated maps, Physica D 62 (1993) 360 371.

[6] R.D. Lee, Malthus and Boserup: a dynamic synthesis, in: The State of Population Theory, D. Coleman and R.S. Schofield, eds. Basil Blackwell, Oxford, 1986, pp. 96-129.

[7] R.D. Lee, Induced population growth and induced technological progress: their interaction in the accelerating stage Mathematical Population Studies 1 (1988) 265-288.

[8] D. Meadows, The Limits to Growth, Universe Books, New York, 1972.

[9] E.F. Mishchenko and N.Kh. Roszow, Differential Equations with Small Parameters and Relaxation Oscillations, Plenum Press, New York and London, 1980.

[10] S. Rinaldi and S. Muratori, Limit cycles in slow-fast forest-pest models, Theoretical Population Biology $41 / 1$ (1992) 2643

[11] S. Rinaldi and S. Muratori, Slow-fast limit cycles in predatorprey models, Ecological Modeling 61 (1992) 287-308.

[12] W.C. Sanderson, Simulation models of economic, demographic, and environmental interactions: are they on a sustainable development path?, working paper WP-92-87, IIASA, Laxenburg, 1992. 
[13] W.C. Sanderson, Simulation models of demographic, economic, and environmental interactions, in: Population - Development Environment. Understanding Their Interactions in Mauritius, W. Lutz, ed., Springer, Berlin, 1994, pp. 33-71.

[14] W.C. Sanderson, Predictability, complexity, and catastrophe in a collapsible model of population, development, and environmental interactions, Mathematical Population Studies 5(3) (1995) 259-279.
[15] S. Schecter, Persistent unstable equilibria and closed orbits of a singularly perturbed system, Journal of Differential Equations 60 (1985) 131-141

[16] P. Szmolyan, Transversal heteroclinic and homoclinic orbits in singular perturbation problems, Journal of Differential Equations 92 (1991) 252-281.

[17] S. Wolfram, Mathematica: a System for doing Mathematics by Computer, Addison-Wesley, New York, 1991. 
